\title{
COMBATE SISTEMÁTICO DE FORMIGAS-CORTADEIRAS COM ISCAS GRANULADAS, EM EUCALIPTAIS COM CULTIVO MÍNIMO ${ }^{1}$
}

\author{
Ronald Zanetti², José Cola Zanuncio ${ }^{3}$, Antônio José Mayhé-Nunes ${ }^{4}$, Alex Giovanny Barros Medeiros ${ }^{5}$ e \\ Alan Souza-Silva ${ }^{2}$
}

\begin{abstract}
RESUMO - Avaliou-se a eficiência do combate sistemático de formigas-cortadeiras em áreas de reforma de eucalipto com cultivo mínimo, na Celulose Nipo-Brasileira S.A., em Belo Oriente, Minas Gerais, de setembro a dezembro de 1996. Os tratamentos consistiram na aplicação de uma isca granulada com sulfluramida $(0,3 \%)$ de forma sistemática, a granel ou com microporta-iscas, na dosagem de $5 \mathrm{~g}$ a cada $6 \mathrm{~m}^{2}$ e $10 \mathrm{~g}$ a cada $12 \mathrm{~m}^{2}$, respectivamente. A mortalidade das colônias de formigas-cortadeiras foi avaliada 30 dias após a aplicação da isca. Foram encontradas até 396,3, 285,2, 59,3, 55,6, 29,6 e 14,8 colônias de Mycocepurus goeldii, Sericomyrmex sp., Acromyrmex subterraneus molestans, Atta spp., Acromyrmex balzani e Acromyrmex niger (Hymenoptera: Formicidae) por hectare, respectivamente. A eficiência da isca granulada no combate sistemático variou com o método empregado e com a espécie de formiga-cortadeira. A maior eficiência foi obtida para A. subterraneus molestans, com 69,2\% de suas colônias mortas com a isca aplicada a granel e 62,5\% com microporta-iscas, o que indica que a distribuição entre dois pontos com isca nos plantios de eucalipto foi maior que a área de forrageamento das formigas-cortadeiras encontradas e, ou, que a dosagem aplicada por ponto foi insuficiente.
\end{abstract}

Palavras-chave: Acromyrmex, Mycocepurus, Sericomyrmex, controle químico, formigas-cortadeiras e iscas formicidas.

\section{SYSTEMATIC CONTROL OF LEAF-CUTTING ANTS IN AREAS WITH EUCALYPTUS STANDS UNDER MINIMUM CULTIVATION SYSTEM}

\begin{abstract}
The efficiency of a systematic application of baits against leaf-cutting ants was evaluated in a eucalypus plantation under a minimum cultivation system, in areas owned by Celulose Nipo-Brasileira S.A. (CENIBRA), in Belo Oriente, Minas Gerais, Brazil, from September to December 1996. Treatments consisted of applying a granulated bait with sulfluramide (0.3\%) in a systematic manner in bulk and plastic bags at a dose of five grams every $6 \mathrm{~m}^{2}$ (T1) and 10 grams at each $12 \mathrm{~m}^{2}$ (T2). Mortality of colonies of leaf-cutting ants was evaluated 30 days after bait application. A. maximum of 396.3; 285.2; 59.3; 55.6; 29.6 and 14.8 colonies of Mycocepurus goeldii, Sericomyrmex sp., Acromyrmex subterraneus molestans, Atta spp., Acromyrmex balzani and Acromyrmex niger (Hymenoptera: Formicidae), respectively, was found per hectare. The efficiency of this granulated bait, applied in a systematic manner, varied with the method and species of leaf-cutting ants. Higher efficiency was obtained for A. subterraneus molestans, with $69.2 \%$ of dead colonies with the bait applied in bulk and $62.5 \%$ when it was applied in plastic bags. This shows that bait distribution in the eucalyptus plantation was performed at a larger spacing than the foraging area of leaf-cutting ants and/or that the dose was insufficient.
\end{abstract}

Key words: Acromyrmex, Mycocepurus, Sericomyrmex, chemical control, leaf-cutting ants.

1 Recebido para publicação em 16.4.2001.

Aceito para publicação em 12.5.2003.

2 Departamento de Entomologia da Universidade Federal de Lavras - UFLA, Caixa Postal 37, 37200-000 Lavras-MG, <zanetti@ufla.br>. ${ }^{3}$ Departamento de Biologia Animal da Universidade Federal de Viçosa - UFV, 36571-000 Viçosa-MG, <zanuncio@ufv.br>. ${ }^{4}$ Universidade Federal Rural do Rio de Janeiro - UFRRJ, 23890-000 Seropédica-RJ. ${ }^{5}$ Celolulose NipoBrasileira S.A., Caixa Postal 691, 35101-970 Belo Oriente-MG. 


\section{INTRODUÇÃO}

Na reforma de áreas reflorestadas com eucalipto são efetuados vários combates às formigas-cortadeiras, especialmente as das espécies dos gêneros Atta e Acromyrmex, que são consideradas as principais pragas de reflorestamentos no Brasil. Os tipos de combate mais comuns são o localizado (aplicação de formicidas diretamente sobre os ninhos) e o sistemático (as iscas formicidas são distribuídas de forma sistemática na área, independentemente da localização dos ninhos das formigas-cortadeiras). Este último método tem sido pouco estudado, apesar de ser prática comum em muitas empresas reflorestadoras brasileiras, em áreas de implantação, de reforma ou de regeneração, para o controle de sauveiros iniciais e, principalmente, de quenquenzeiros, que podem provocar danos severos à brotação ou às mudas recém-plantadas (Oliveira et al., 1993).

Muitas empresas reflorestadoras passaram a utilizar o cultivo mínimo em seus plantios de eucalipto, visando a redução dos custos na implantação da floresta e a melhoria das condições ambientais dessas áreas, com a eliminação do uso do fogo. Entretanto, tal prática tem dificultado o controle de pragas como as formigascortadeiras, principalmente do gênero Acromyrmex (quenquéns). Estas espécies apresentam colônias menores e mais abundantes que as das saúvas e são difíceis de ser localizadas sob a vegetação e restos de colheita florestal, o que contribui para aumentar a sua importância e a necessidade de pesquisas que visam o seu combate em áreas reflorestadas.

No Brasil, testes com iscas à base de sulfluramida para o controle de formigas-cortadeiras apresentaram excelentes resultados para muitas espécies de saúvas, incluindo Atta laevigata (Della Lucia et al., 1992; Zanuncio et al., 1992; Alves et al., 1996, 1997), Atta sexdens rubropilosa (Della Lucia et al., 1992; Laranjeiro \& Zanuncio, 1995; Zanuncio et al., 1997; Lopes et al., 1999), Atta sexdens sexdens (Cruz et al., 1996). Este princípio ativo mostrou, também, alta eficiência no controle de quenquéns, como verificado por Cruz et al. (2000), para Acromyrmex octospinosus, e por Zanuncio et al. (1996), para Acromyrmex subterraneus molestans, quando foi verificada eficiência de $100 \%$ após 30 dias da aplicação. Com base neste resultado, os autores sugeriram que as avaliações de formicidas para o controle de quenquéns sejam feitas com esse intervalo de tempo, para evitar a inclusão do efeito da migração e da mortalidade

R. Árvore, Viçosa-MG, v.27, n.3, p.387-392, 2003 natural no conjunto dos dados, o que levaria a erros de interpretação dos resultados.

Dessa forma, o presente trabalho teve como objetivo testar a eficiência de uma isca formicida à base de sulfluramida $(0,3 \%)$ aplicada de forma sistemática, a granel ou com microporta-iscas, no controle das principais espécies de formigas-cortadeiras presentes em uma área de reforma de eucalipto com cultivo mínimo no município de Belo Oriente, Minas Gerais.

\section{MATERIAL E MÉTODOS}

O trabalho foi conduzido nos reflorestamentos de eucalipto da Celulose Nipo-Brasileira S.A., em Belo Oriente, Minas Gerais, de setembro a dezembro de 1996, em três áreas de 18 ha cada, onde o eucalipto havia sido colhido e os sauveiros combatidos com termonebulização 60 dias antes do início do experimento, de modo que restavam apenas sauveiros iniciais, quenquenzeiros e colônias de outros Attini.

Em cada área foram marcadas dez parcelas de $270 \mathrm{~m}^{2}(9 \times 30 \mathrm{~m})$, que receberam um dos seguintes tratamentos: $\mathrm{T} 1$ = aplicação sistemática a granel de isca à base de sulfluramida $(0,3 \%)$ na dosagem de $5 \mathrm{~g}$ a cada $6 \mathrm{~m}^{2}(3 \times 2 \mathrm{~m}) ; \mathrm{T} 2$ = aplicação sistemática a granel de isca à base de sulfluramida $(0,3 \%)$ em microporta-iscas (saquinhos de polietileno) de $10 \mathrm{~g}$ a cada $12 \mathrm{~m}^{2}(3 \times 4 \mathrm{~m})$; e T3 = testemunha (sem aplicação de isca formicida). A isca a granel foi aplicada com dosador-aplicador costal da marca Bombata. Trinta dias após a aplicação das iscas, os formigueiros foram abertos, seguindo-se o procedimento descrito por Zanuncio et al. (1996).

As colônias de formigas-cortadeiras, nas áreas estudadas, foram contadas e identificadas, antes da aplicação dos tratamentos. Além disto, avaliou-se a mortalidade dos formigueiros após 30 dias da aplicação da isca formicida. Os dados foram corrigidos para raiz quadrada de $(x+0,5)$, antes de serem submetidos à análise de variância e ao teste de agrupamento de médias de ScottKnott, a 5\% de significância (Scott \& Knott, 1974). A eficiência dos tratamentos foi comparada pela fórmula de Schneider \& Orelli (Nakano et al., 1981).

\section{RESULTADOS E DISCUSSÃO}

Nas áreas estudadas foram encontrados formigueiros de Attini das espécies Mycocepurus goeldii Forel, Sericomyrmex sp., Acromyrmex subterraneus molestans 
Santschi, Atta sexdens rubropilosa Forel, Acromyrmex balzani Emery e Acromyrmex niger (Fr. Smith) com até 396,3, 285,2, 59,3, 55,6, 29,6 e 14,8 colônias/ha, respectivamente (Quadro 1). A primeira espécie foi mais abundante, seguida por Sericomyrmex sp. e pelas demais espécies, que tiveram densidade semelhante entre si em todos os tratamentos. Independentemente da espécie, não houve diferença no número total de ninhos de formigas por hectare na contagem feita antes da aplicação dos tratamentos, possivelmente por tratar-se de áreas bastante homogêneas quanto ao tipo de solo, relevo, clima, manejo florestal, entre outros. Tal fato facilitou a interpretação dos dados.

M. goeldii apresentou maior número de colônias que as demais formigas no tratamento com a isca aplicada a granel (396,3/ha). Duas espécies, M. goeldii (166,7 colônias/ha) e Sericomyrmex sp. (285,2 colônias/ha), foram predominantes na área que recebeu microportaiscas, enquanto na testemunha apenas a primeira apresentou maior número de colônias/ha $(229,6)$ que as demais. Esses dados corroboram os de Pacheco \& Berti Filho (1987), que encontraram altas infestações dessas espécies de formigas em plantios de eucalipto, embora com baixo nível de dano, pelo corte de gemas iniciais durante a rebrota das plantas. Entretanto, a literatura cita que, potencialmente, M. goeldii e Sericomyrmex sp. podem representar problemas para mudas ou rebrota de eucalipto (Gonçalves, 1975; Pacheco \& Berti Filho, 1987).
A. niger, A. balzani e A. subterraneus molestans foram menos freqüentes e menos abundantes (Quadro 1), apesar de fazerem parte do grupo das quenquéns mais daninhas e comuns em plantios de eucalipto no Estado de Minas Gerais. A. niger merece preocupação, pois Pacheco \& Berti Filho (1987) relataram atividade intensa de corte de folhas de eucalipto por essas formigas, em Minas Gerais e São Paulo. Os autores encontraram muitas árvores de Eucalyptus saligna com ponteiros totalmente desfolhados por essa formiga.

A mortalidade das colônias de formigas-cortadeiras foi, em geral, baixa nos diversos tratamentos. A maior eficiência foi observada na área com microporta-iscas $(26,4)$, seguida da que recebeu isca a granel $(25,4 \%)$, enquanto a testemunha (sem aplicação de inseticida) apresentou mortalidade natural de 3,6\% (Quadro 2). A maior eficiência dessa isca foi registrada para A. subterraneus molestans, com mortalidade de $69,2 \mathrm{e}$ de $62,5 \%$, maior que a registrada para $A$. balzani $(25,0 \mathrm{e}$ $25,0 \%)$ e $M$. goeldii $(22,4$ e 20,0\%), nos tratamentos a granel e com microporta-iscas, respectivamente. A melhor eficiência da isca granulada para A. subterraneus molestans é de grande importância, pois esta é considerada a principal espécie-praga do gênero Acromyrmex para o setor florestal, em áreas de plantio de eucalipto (Zanuncio et al., 1996). Além disto, a espécie destaca-se por apresentar ninhos pequenos, porém em maior número que os da Atta spp., o que a leva a causar danos acentuados em plantios de eucalipto.

Quadro 1 - Número de colonias de formigas-cortadeiras por hectare (média \pm erro-padrão) em áreas de cultivo mínimo que receberam combate com iscas formicidas aplicadas a granel (granel) e com microporta-iscas (porta-iscas) e sem aplicação de isca (testemunha). Município de Belo Oriente, Estado de Minas Gerais, 1996

Table 1 - Number of leaf-cutting ants colonies per hectare (mean \pm standard error) in controlled areas with baits applied without and inside plastic bags,compared to treatment without baits (control). Belo Oriente, Minas Gerais, Brazil, 1996

\begin{tabular}{|l|c|c|c|}
\hline \multirow{2}{*}{ Espécie de Formiga } & \multicolumn{3}{|c|}{ Número de Colônias de Formigas/ha* } \\
\cline { 2 - 4 } & Granel & Porta-isca & Testemunha \\
\hline Acromyrmex balzani & $29,6 \pm 15,4 \mathrm{aC}$ & $14,8 \pm 6,0 \mathrm{aB}$ & $29,6 \pm 17,3 \mathrm{aB}$ \\
Acromyrmex niger & $0,0 \pm 0,0 \mathrm{aC}$ & $11,1 \pm 5,6 \mathrm{aB}$ & $14,8 \pm 9,9 \mathrm{aB}$ \\
Acromyrmex subterraneus molestans & $48,1 \pm 20,7 \mathrm{aC}$ & $29,6 \pm 19 \mathrm{aB}$ & $59,3 \pm 14,8 \mathrm{aB}$ \\
Atta sp. & $55,6 \pm 32,8 \mathrm{aC}$ & $7,4 \pm 4,9 \mathrm{aB}$ & $55,5 \pm 34,1 \mathrm{aB}$ \\
Mycocepurus goeldii & $396,3 \pm 93,2 \mathrm{aA}$ & $166,7 \pm 35,9 \mathrm{bA}$ & $229,6 \pm 51,7 \mathrm{bA}$ \\
Sericomyrmex sp. & $144,4 \pm 45,3 \mathrm{aB}$ & $285,2 \pm 65,9 \mathrm{aA}$ & $40,7 \pm 29,4 \mathrm{bB}$ \\
\hline Média geral & $122,3 \mathrm{a}$ & $85,8 \mathrm{a}$ & $71,6 \mathrm{a}$ \\
\hline
\end{tabular}

* Médias seguidas pela mesma letra minúscula na linha e maiúscula na coluna não diferem estatisticamente entre si (Scott-Knott, $\mathrm{p}<0,05)$. 
A baixa eficiência da isca para as demais espécies de formigas-cortadeiras pode ser devido à dificuldade de carregamento de seus grânulos por operárias dessas formigas. Além disto, a distribuição de iscas em distâncias maiores que as de forrageamento das formigas-cortadeiras e a sua dificuldade em cortar o saco plástico são aspectos que também devem ser considerados para explicar a menor eficiência de controle da isca aplicada. A hipótese da maior distância entre os pontos de distribuição da isca, em relação à área de forrageamento das formigascortadeiras, fica mais evidente pelo fato de ter sido obtida maior eficiência para A. subterraneus molestans, que é a espécie de formiga desse gênero com ninhos maiores entre as espécies encontradas e que, possivelmente, deve apresentar maiores áreas de forrageamento. Além disto, tal fato pode ser reforçado pela alta eficiência de iscas à base de sulfluramida contra essa formiga-cortadeira em trabalho de Zanuncio et al. (1996) e contra espécies do mesmo gênero, como Acromyrmex laticeps nigrosetosus (Zanuncio et al., 1999) e Acromyrmex octospinosus (Camargo et al., 1997), quando a isca foi aplicada diretamente nos ninhos dessas formigas. $\mathrm{O}$ aspecto relacionado à granulometria das iscas formicidas deve ser considerado no seu desenvolvimento, de maneira que possam ser carregadas pela maioria das formigas-praga, sem maiores dificuldades. Algumas empresas florestais estão macerando a isca granulada ou aplicando seu resíduo em pó, obtido nas empresas produtoras, para o controle de M. goeldii e Sericomyrmex sp., por se tratarem de formigas pequenas e que, por isto, apresentam dificuldade em transportar os grânulos de iscas formicidas. A dificuldade das operárias de formigas-cortadeiras em cortar o plástico do microporta-iscas também pode ser uma possível causa da baixa eficiência para as formigas de menor tamanho, o que mostra a necessidade de desenvolver embalagens mais adequadas para serem utilizadas em distribuição sistemática contra essas espécies de formigas-cortadeiras. Finalmente, acredita-se que M. goeldii e Sericomyrmex sp. tem área de forrageamento muito restrita ao redor de seus ninhos, o que torna necessário a distribuição da isca de forma tão adensada dificultando o seu uso em distribuição sistemática para o controle dessas espécies de formigascortadeiras em áreas de reflorestamentos.

Outro fator que deve ser considerado é que a menor eficiência da isca nos tratamentos testados, em contraste com os resultados de pesquisas anteriores, pode estar associada ao fato de a área de estudo ser de cultivo mínimo, onde havia cobertura muito intensa de gramíneas e ervas daninhas, que dificultariam a localização e o transporte da isca e poderiam reduzir o seu transporte por essas formigas.

É interessante registrar que a testemunha apresentou mortalidade natural de $18,1 \%$ para $A$. subterraneus molestans (Quadro 2). Zanuncio et al. (1996), avaliando diferentes dosagens de iscas para o controle dessa espécie, encontraram mortalidade natural muito semelhante, de $18,75 \%$. Os autores levantaram a hipótese de que A. subterraneus molestans apresenta hábito migratório em curto período de tempo (30 dias), em razão do tipo de nidificação superficial dessa quenquém, o que poderia ter contribuído para superestimar a mortalidade natural

Quadro 2 - Porcentagem de eficiência do combate de formigas-cortadeiras com iscas formicidas aplicadas a granel (granel) e com microporta-iscas (porta-iscas) e porcentagem de mortalidade natural (testemunha). Município de Belo Oriente, Estado de Minas Gerais, 1996

Table 2 - Efficiency percentage of baits applied without and inside plastic bags compared to treatment without baits (control). Belo Oriente, Minas Gerais, Brazil, 1996

\begin{tabular}{|c|c|c|c|c|}
\hline \multirow{2}{*}{ Espécie de Formiga } & \multicolumn{4}{|c|}{ Eficiência (\%) } \\
\hline & Granel & Porta-isca & Testemunha & Média \\
\hline Acromyrmex balzani & 25,0 & 25,0 & 0,0 & 16,7 \\
\hline Acromyrmex niger & 0,0 & 33,3 & 0,0 & 11,1 \\
\hline Acromyrmex subterraneus molestans & 69,2 & 62,5 & 18,1 & 49,9 \\
\hline Atta sp. & 13,3 & 6,7 & 0,0 & 6,7 \\
\hline Mycocepurus goeldii & 22,4 & 20,0 & 3,2 & 15,2 \\
\hline Sericomyrmex sp. & 23,1 & 10,7 & 0,0 & 11,3 \\
\hline Média geral & 25,5 & 26,4 & 3,6 & 18,5 \\
\hline
\end{tabular}

R. Árvore, Viçosa-MG, v.27, n.3, p.387-392, 2003 
encontrada. Por este motivo, os autores recomendaram que a avaliação da eficiência de iscas formicidas para quenquéns seja feita aos 30 dias, evitando-se assim a inclusão de um fator extra de variação dos dados. Portanto, é possível que tal fato ocorra com outras espécies de Acromyrmex, como Acromyrmex aspersus, Acromyrmex crassispinus e Acromyrmex subterraneus brunneus, que também constroem ninhos superficiais. Por isto, a mortalidade natural de ninhos dessas formigascortadeiras deve ser quantificada e usada como um fator de correção para determinação da eficiência de iscas formicidas.

Pelos motivos citados, e considerando que o cultivo mínimo é uma realidade no setor florestal, essas questões merecem ser investigadas em futuros trabalhos nesta linha de pesquisa, pois essa isca tem apresentado eficiência muito alta contra formigas-cortadeiras em aplicação localizada, tanto em dosagem única (Laranjeiro \& Zanuncio, 1995; Lopes et al., 1999), como com dose por metro quadrado de formigueiro (Cruz et al., 2000; Zanuncio et al., 1997).

\section{CONCLUSÕES}

1. Mycocepurus goeldii foi mais abundante que as demais espécies de Attini encontradas em plantios de eucalipto na região de Belo Oriente, Minas Gerais.

2. A eficiência do combate foi maior para $A$. subterraneus molestans, tanto na aplicação a granel quanto na com microporta-isca.

3. A eficiência para as demais espécies de formigascortadeiras foi baixa, indicando que devem ser desenvolvidos trabalhos que visam melhorar a eficiência de controle dessas espécies de formigascortadeira através da distribuição mais adensada de isca, o que facilitaria sua localização por essas espécies de formigas-cortadeiras.

\section{AGRADECIMENTO}

Ao Conselho Nacional de Desenvolvimento Científico e Tecnológico $(\mathrm{CNPq})$ e à Fundação de Amparo à Pesquisa do Estado de Minas Gerais (FAPEMIG), pelas bolsas e pelo auxílio. À Celulose Nipo-Brasileira S.A. (CENIBRA), pelo apoio para realização desta pesquisa. Ao professor Evaldo Ferreira Vilela, pelas sugestões ao manuscrito.

\section{REFERÊNCIAS BIBLIOGRÁFICAS}

ALVES, J. B. et al. Métodos de distribuição de isca granulada em formigueiros de Atta laevigata (F. Smith). Revista Árvore, v. 20, n. 1, p. 111-116, 1996.

ALVES, J. B. et al. Paralisação de forrageamento e controle de Atta laevigata (F. Smith) (Hymenoptera: Formicidae) com Mirex-S (Sulfluramida) em duas metodologias de medição de formigueiros. Revista Árvore, v. 21, n. 1, p. 141-146, 1997.

CAMARGO, F. R. A. et al. Control de Acromyrmex crassipinus (Hymenoptera: Formicidae) en areas de rebrote de Eucalyptus grandis com un sebo a base de sulfluramida. Revista Yvyrareta, n. 8, p. 71-74, 1997.

CRUZ, A. P. et al. Eficiência de iscas formicidas à base de sulfluramida e de clorpirifós no controle de Atta sexdens sexdens (Hymenoptera, Formicidae), no trópico úmido. Acta Amazônica, v. 26, n. 3, p. 145-150, 1996.

CRUZ, A. P. et al. Eficiência de cebos granulados a base de sulfluramida o de clorpirifós en el control de Acromyrmex octospinosus (Hymenoptera: Formicidae) en el trópico húmedo. Revista Colombiana de Entomologia, v. 26, n. 1/ 2, p. 67-69, 2000.

DELLA LUCIA, T. M. C. et al. Aceitação de iscas granuladas com sulfluramida, novo principio ativo, por formigas cortadeiras, no campo. Revista Árvore, v. 16, n. 2, p. 218-223, 1992.

GONÇALVES, C. R. Formigas dos gêneros Sericomyrmex e Trachymyrmex cortando folhas verdes de plantas (Hymenoptera., Formicidae, Myrmicinae, Attini). In: REUNIÃO ANUAL DA SBPC, 28, 1975, Belo Horizonte. Anais... Belo Horizonte: 1975. p. 1670.

LARANJEIRO, A. J.; ZANUNCIO, J. C. Avaliação da isca à base de sulfluramida no controle de Atta sexdens rubropilosa pelo processo dosagem única de aplicação. IPEF, v. 48/49, n. 1, p. 144-152, 1995.

LOPES, E. T. et al. Efeito do tamanho de formigueiros de Atta sexdens rubropilosa Forel, 1908 (Hymenoptera: Formicidae) na dosagem de iscas granuladas por olheiro ativo. Revista Árvore, v. 23, n. 4, p. 473-478, 1999.

NAKANO, O.; SILVEIRA NETO, S.; ZUCCHI, R. A. Entomologia Econômica. Piracicaba: Livro Ceres, 1981. $303 \mathrm{p}$.

R. Árvore, Viçosa-MG, v.27, n.3, p.387-392, 2003 
OLIVEIRA, A. C. et al. Um estudo de caso: o sistema de monitoramento e controle de formigas cortadeiras na Mannesmann Fi-El Florestal Ltda. In: DELLA LUCIA, T. M. C. (Ed.s). As formigas cortadeiras. Viçosa: 1993. p. 242-255.

PACHECO, P.; BERTI FILHO, E. Formigas Quenquéns. In: PACHECO, P., BERTI FILHO, E. (Eds.). Formigas cortadeiras e o seu controle. Piracicaba, 1987. p. 3-17.

SCOTT, A. J.; KNOTT, M. A. A cluster analysis method for grouping means in the analysis of variance. Biometrics, v. 30, p. 507-512, 1974.

ZANUNCIO, J. C. et al. Eficiência da isca granulada à base de sulfluramida no controle da formiga cortadeira Atta laevigata (F. Smith, 1858) (Hymenoptera: Formicidae). Revista Árvore, v. 16, n. 3, p. 357-361, 1992.
ZANUNCIO, J. C.; LARANJEIRO, A. J.; DeSOUZA, O. Controle de Acromyrmex subterraneus molestans Santschi (Hymenoptera: Formicidae) com sulfluramida. Anais da Sociedade Entomológica do Brasil, v. 25, n. 3, p. 383-388, 1996.

ZANUNCIO, J. C. et al. Uso da isca granulada com sulfluramida (0,3\%), no controle de Atta sexdens rubropilosa Forel, 1908 (Hymenoptera: Formicidae). Cerne, v. 3, n. 1, p. 161-169, 1997.

ZANUNCIO, J. C. et al. Controle de Acromyrmex laticeps nigrosetosus (Hymenoptera: Formicidae), em eucaliptal no Pará, com iscas granuladas com sulfluramida ou clorpirifós. Acta Amazonica, v. 29, n. 4, p. 639-645, 1999. 